\title{
Notification that New Names and New Combinations Have Appeared in Volume 46, No. 2, of the IJSB $^{a}$
}

\begin{tabular}{|c|c|c|c|}
\hline Name: & Proposed as: & Authors: & $\begin{array}{l}\text { IJSB reference } \\
\text { description: }\end{array}$ \\
\hline Lactobacillus sake & emend. & Klein et al. & $46(2): 374$ \\
\hline Lactobacillus curvatus & emend. & Klein et al. & $46(2): 375$ \\
\hline Sulfolobus hakonensis & sp. nov. & Takayanagi et al. & $46(2): 381$ \\
\hline Thermoanaerobacterium & emend. & Liu et al. & $46(2): 394$ \\
\hline Thermoanaerobacterium aotearoense & sp. nov. & Liu et al. & $46(2): 395$ \\
\hline Desulfotomaculum thermocisternum & sp. nov. & Nilsen et al. & $46(2): 401$ \\
\hline Thermus oshimai & sp. nov. & Williams et al. & 46(2):406 \\
\hline Thiobacillus thermosulfatus & sp. nov. & Shooner et al. & $46(2): 414$ \\
\hline Thermothrix azorensis & sp. nov. & Odintsova et al. & $46(2): 426$ \\
\hline Tsukamurella pulmonis & sp. nov. & Yassin et al. & $46(2): 434$ \\
\hline Mycoplasma elephantis & sp. nov. & Kirchhoff et al. & $46(2): 440$ \\
\hline Desulfitobacterium hafniense & sp. nov. & Christiansen and Ahring & $46(2): 446$ \\
\hline Lactobacillus panis & sp. nov. & Wiese et al. & $46(2): 452$ \\
\hline Clostridium acetireducens & sp. nov. & Örlygsson et al. & $46(2): 458$ \\
\hline Rubrobacter xylanophilus & sp. nov. & Carreto et al. & $46(2): 463$ \\
\hline Bacillus vallismortis & sp. nov. & Roberts et al. & $46(2): 474$ \\
\hline Vibrio tapetis & sp. nov. & Borrego et al. & $46(2): 483$ \\
\hline Halobacillus & gen. nov. & Spring et al. & $46(2): 495$ \\
\hline Halobacillus litoralis & sp. nov. & Spring et al. & $46(2): 495$ \\
\hline Halobacillus trueperi & sp. nov. & Spring et al. & $46(2): 495$ \\
\hline Halobacillus halophilus (basonym Sporosarcina halophila) & comb. nov. & Spring et al. & $46(2): 495$ \\
\hline Caloramator indicus & sp. nov. & Chrisostomos et al. & $46(2): 500$ \\
\hline Sporobacter & gen. nov. & Grech-Mora et al. & $46(2): 517$ \\
\hline Sporobacter termitidis & sp. nov. & Grech-Mora et al. & 46(2):517 \\
\hline Microsphaera & gen. nov. & Yoshimi et al. & $46(2): 524$ \\
\hline Microsphaera multipartita & sp. nov. & Yoshimi et al. & $46(2): 524$ \\
\hline Sarcobium pro synon., Legionella & synon. & Hookey et al. & $46(2): 529$ \\
\hline Legionella lytica (basonym Sarcobium lyticum) & comb. nov. & Hookey et al. & $46(2): 529$ \\
\hline Bacillus thermoaerophilus & sp. nov. & Meier-Stauffer et al. & 46(2):540 \\
\hline Deleya pro synon., Halomonas & synon. & Dobson and Franzmann & $46(2): 556$ \\
\hline Halovibrio pro synon., Halomonas & synon. & Dobson and Franzmann & 46(2):556 \\
\hline Halomonas aquamarina (basonym Deleya aquamarina) & comb. nov. & Dobson and Franzmann & $46(2): 556$ \\
\hline Halomonas variabilis (basonym Halovibrio variabilis) & comb. nov. & Dobson and Franzmann & 46(2):556 \\
\hline Halomonas venusta (basonym Deleya venusta) & comb. nov. & Dobson and Franzmann & 46(2):556 \\
\hline Halomonas halodenitrificans (basonym Paracoccus halodenitrificans) & comb. nov. & Dobson and Franzmann & $46(2): 556$ \\
\hline Halomonas cupida (basonym Deleya cupida) & comb. nov. & Dobson and Franzmann & 46(2):556 \\
\hline Halomonas pacifica (basonym Deleya pacifica) & comb. nov. & Dobson and Franzmann & $46(2): 556$ \\
\hline Halomonas marina (basonym Deleya marina) & comb. nov. & Dobson and Franzmann & $46(2): 556$ \\
\hline Halomonas halophila (basonym Deleya halophila) & comb. nov. & Dobson and Franzmann & 46(2):556 \\
\hline Halomonas salina (basonym Deleya salina) & comb. nov. & Dobson and Franzmann & 46(2):556 \\
\hline Halomonas & emend. & Dobson and Franzmann & $46(2): 557$ \\
\hline Halomonadaceae & emend. ${ }^{b}$ & Dobson and Franzmann & 46(2):558 \\
\hline Pseudobutyrivibrio & gen. nov. & van Gylswyk et al. & $46(2): 561$ \\
\hline Pseudobutyrivibrio ruminis & sp. nov. & van Gylswyk et al. & $46(2): 561$ \\
\hline Bifidobacterium inopinatum & sp. nov. & Crociani et al. & $46(2): 569$ \\
\hline Bifidobacterium denticolens & sp. nov. & Crociani et al. & $46(2): 570$ \\
\hline Lactobacillus paraplantarum & sp. nov. & Curk et al. & $46(2): 598$ \\
\hline Cytophaga & emend. ${ }^{c}$ & Nakagawa and Yamasato & 46(2):600 \\
\hline Marinilabilia & gen. nov. & Nakagawa and Yamasato & $46(2): 600$ \\
\hline Marinilabilia salmonicolor (basonym Cytophaga salmonicolor) & comb. nov. & Nakagawa and Yamasato & 46(2):600 \\
\hline Marinilabilia agarovorans (basonym Cytophaga agarovorans) & comb. nov. & Nakagawa and Yamasato & 46(2):600 \\
\hline Meiothermus & gen. nov. & Nobre et al. & $46(2): 605$ \\
\hline Meiothermus ruber (basonym Thermus ruber) & comb. nov. & Nobre et al. & $46(2): 605$ \\
\hline Meiothermus silvanus (basonym Thermus silvanus) & comb. nov. & Nobre et al. & $46(2): 605$ \\
\hline Meiothermus chliarophilus (basonym Thermus chliarophilus) & comb. nov. & Nobre et al. & $46(2): 605$ \\
\hline Thermus & emend. & Nobre et al. & $46(2): 605$ \\
\hline Rickettsia africae & sp. nov. & Kelly et al. & $46(2): 612$ \\
\hline
\end{tabular}

${ }^{a}$ This listing of names published in a previous issue of IJSB is provided as a service to bacteriology to assist in the recognition of new names and new descriptions. This procedure was proposed by the Judicial Commission [Minute II (ii), Int. J. Syst. Bacteriol. 41:185, 1991]. The names given herein have priority according to the issue of the IJSB in which they were published.

${ }^{b}$ The emended description of the family Halomonadaceae comprises the genera Halomonas, Chromohalobacter, and Zymobacter.

${ }^{c}$ The emended description is restricted to Cytophaga hutchinsonii, the type species of the genus, and to Cytophaga aurantiaca. 